\title{
Las transiciones a la democracia en España y Portugal como pasados difíciles. Representaciones sociales del profesorado de Educación Secundaria en formación ${ }^{1}$
}

\author{
José ARMAS CASTRO \\ Jorge CONDE MIGUÉLEZ \\ Cristina MAIA \\ Ana RITA FÉRIAS
}

Datos de contacto:

José Armas Castro

Universidad de Santiago de

Compostela

xose.armas@usc.es

Jorge Conde Miguélez

Universidad de Santiago de

Compostela

jorge.conde@usc.es

Cristina Maia

Escola Superior De Educação do

Politécnico do Porto

cristinamaia@ese.ipp.pt

Ana Rita Férias

Universidade de Santiago de

Compostela e Escola Superior de

Educação do Politécnico do Porto

ana.ferias@hotmail.com

Recibido: 14/07/2020

Aceptado: 17/02/2021

\section{RESUMEN}

Se ofrecen resultados de una investigación cualitativa de corte fenomenológico sobre las representaciones de una muestra de profesorado español y portugués de educación secundaria en formación sobre la enseñanza de las transiciones de la dictadura a la democracia en España y Portugal. En la investigación participaron 39 profesores de educación secundaria en formación que cursaban sus estudios en las universidades de Santiago de Compostela (24) y Porto (15). La información se recogió por medio de un cuestionario con ítems abiertos y otros de escala Likert en el que se les solicitaban personajes y acontecimientos relevantes de las transiciones en ambos países, una selección de ideas clave para llevar a las aulas de educación secundaria los procesos de transición, y el grado de acuerdo con cuatro actitudes del profesorado a la hora de enseñar pasados difíciles como las transiciones dictadurademocracia. Los resultados indican que el núcleo central de las representaciones del profesorado en formación está formado por personajes y acontecimientos emblemáticos con una importante presencia en los medios y en las industrias culturales; las narrativas de los profesores sobre las transiciones participan fundamentalmente de las tipologías "memoria colectiva"” y "disciplinar"; las actitudes que tienen más aceptación entre los futuros profesores son "imparcialidad neutral" e "imparcialidad comprometida".

PALABRAS CLAVE: Transiciones a la democracia; Educación histórica; Formación de profesores; Educación secundaria; Representaciones sociales.

\footnotetext{
${ }^{1}$ Este trabajo forma parte de la investigación financiada por el Plan Nacional de l+D+i del MINECO (PGC2018-094491-B-C31) y cofinanciada con fondos FEDER de la UE.
} 


\title{
Transitions to democracy in Spain and Portugal as Difficult Pasts. Social Representations of Secondary Education Preservice Teachers
}

\begin{abstract}
Results of a qualitative research of phenomenological type are offered on the representations and attitudes of a sample of Spanish and Portuguese preservice teachers of secondary education related to the teaching of the transitions from dictatorship to democracy in Spain and Portugal. The research involved 39 preservice teachers of secondary education who were studying at the universities of Santiago de Compostela (24) and Porto (15). Information was collected by means of a questionnaire with open response and Likert scale items. Participants were asked for relevant characters and events of the transitions in both countries, a selection of key ideas to take to the secondary classrooms, and the degree of agreement with four different attitudes of teachers when teaching difficult pasts such as the dictatorshipdemocracy transitions. The results indicate that the central nucleus of training teachers' representations is made up of emblematic characters and events with an important presence in the media and in the cultural industries; the teachers' narratives about transitions participate fundamentally in the typologies "collective memory" and "disciplinary"; the most popular attitudes among training teachers are "neutral impartiality" and "committed impartiality".
\end{abstract}

KEYWORDS: Transitions to democracy; History education; Teachers preparation; Secondary education; Social representations.

\section{Introducción}

Los procesos de democratización de las últimas décadas del siglo XX en el Sur de Europa y en Latinoamérica, han convertido las transiciones de dictaduras a sistemas democráticos en objeto de estudio, en primer lugar, por parte de sociólogos y politólogos, hasta el punto de dar lugar a una rama específica de estudio: "la transitología" (Schmitter y Lynn, 1994). Algo más tarde también los historiadores prestaron atención a las transiciones a la democracia. Si los estudios más tradicionales se han centrado en los acontecimientos políticos, los personajes que los protagonizaron o las instituciones democráticas fundadas, contribuyendo así a una cierta mitificación de estos procesos, la historiografía más actual se ha ocupado de analizar los diferentes contextos sociales y políticos (Loff, 2004; Soto, 2012; Ysàs, 2010), de comparar diferentes procesos de transición (Feria, 2018; Gómez, 2011; Lemus, 2012), o de explorar nuevos temas como los mitos, la violencia o la memoria de las transiciones a la democracia (Baby, Compagnon y 
González, 2009; Chaput y Pérez, 2015; Izquierdo, 2018; Loff, Soutelo y Piedade, 2014; Varela, 2012).

La presencia en las aulas de las transiciones dictadura-democracia se ha producido lentamente y no sin controversias en los países que experimentaron estas transformaciones. Las transiciones en España, Portugal, Argentina o Chile reúnen varios rasgos característicos de los "pasados difíciles" (Gross y Terra, 2019): ocupan un lugar central en las historias nacionales, ponen en cuestión versiones comúnmente aceptadas del pasado, conectan con problemas que generan discusión en el presente, e implican acontecimientos de violencia colectiva y/o estatal. En este sentido, las transiciones más o menos violentas comparten con temáticas como las guerras, la esclavitud, el holocausto o el exterminio de comunidades indígenas la perspectiva de la enseñanza y aprendizaje de "pasados difíciles" (Epstein y Peck, 2018; Gross y Terra, 2019). Abordar la enseñanza de historias controvertidas en la formación inicial de profesores puede ayudarles a revisar creencias de sentido común, vencer resistencias hacia estas temáticas y contrastar estrategias de enseñanza eficaces (Barton, 2019; Goldberg y Savenije, 2018).

La enseñanza y aprendizaje de la transición a la democracia en España y Chile ha sido objeto de un proyecto de investigación (TRADDEC) que se ha ocupado, tanto de las representaciones y prácticas del profesorado (Martínez, 2013; Martínez, Muñoz y Sánchez, 2019), como de los aprendizajes de los estudiantes (Sánchez, Martínez, Miguel y López, 2019; Vásquez, Sánchez, y Vásquez, 2018) o su tratamiento en los currículos y en los manuales escolares (Aceituno, 2011; Martínez, 2011). Otros trabajos se han ocupado de las prácticas de profesores y estudiantes en las aulas al estudiar la Transición (Santiago, 2014) o del trabajo con las memorias de los alumnos (Molpeceres, 2011).

La revolución portuguesa, la transición a la democracia y sus memorias han suscitado importantes debates entre historiadores, en la sociedad y en el contexto educativo. Algunos trabajos han puesto de manifiesto el lugar que la dictadura de Salazar o la Revolución del 25 de abril ocupan en la construcción de la conciencia histórica de los estudiantes (Armas, Moreira, Maia y Conde, 2019; Barca y Schmidt, 2013), mientras que otros estudian las ideas de los alumnos sobre pasados dolorosos que dejan marcas en los procesos de reconciliación posteriores a conflictos, como la guerra colonial (Alves, Ribeiro, Oliveira y Moreira, 2012) o la dictadura salazarista y la revolución (Férias, Armas y Maia, 2019).

La teoría de las representaciones sociales (Moscovici, 2000; Sammut, Andreouli, Gaskell y Valsiner, 2015) ha demostrado ser una herramienta conceptual valiosa para interpretar la forma en que las personas atribuyen significado a diferentes realidades sociales. Desde el ámbito de la psicología social, los estudios sobre representaciones sociales se han difundido a otros como la salud, el medio ambiente, la política y, para lo que aquí nos interesa, la educación (Castorina y Barreiro, 2012; Jodelet, 2011). En nuestro caso, la teoría de las representaciones sociales servirá de lente para observar las perspectivas de 
profesores en formación sobre la enseñanza de las transiciones de la dictadura a la democracia en España y Portugal.

Este trabajo pretende responder a los siguientes objetivos: a) analizar las representaciones de una muestra de profesores de educación secundaria en formación sobre personajes y acontecimientos relevantes de las transiciones de la dictadura a la democracia en España y Portugal; b) caracterizar las narrativas que los profesores en formación pretenden trasladar a las aulas de secundaria sobre las transiciones; c) analizar las opciones de los futuros profesores respecto a cuatro perspectivas sobre la enseñanza de los pasados difíciles (Kelly, 1986): neutralidad excluyente, parcialidad excluyente, imparcialidad neutral e imparcialidad comprometida.

\section{Método}

Este estudio se inspira en los principios de la investigación cualitativa (Denzin y Lincoln, 2018; Flick, 2015), más concretamente en la tradición fenomenológica, en la medida en que trata de explicar una realidad educativa concreta teniendo en cuenta los puntos de vista de los participantes.

En la investigación participaron 39 profesores de educación secundaria en formación, 24 españoles y 15 portugueses. Los españoles eran estudiantes del Máster en Profesorado de Educación Secundaria que cursaban la especialidad de Ciencias Sociales y Humanidades en la Universidad de Santiago de Compostela. Los portugueses seguían el Máster en Enseñanza de Historia y Geografía en el Tercer Ciclo de Educación Básica y Educación Secundaria de la Universidad de Porto. Ambas modalidades de formación son las que habilitan para el acceso a la docencia en el nivel de educación secundaria en España y Portugal. La distribución por género era bastante equilibrada, 17 mujeres y 22 hombres, siendo la media de edad 25,9 años.

La recogida de datos se realizó entre marzo y mayo de 2020 en el contexto de aula del Máster en Profesorado de Educación Secundaria de la Universidad de Santiago de Compostela, mientras que con el alumnado de la Universidad de Porto se utilizó un formulario online (Google forms) debido a la suspensión de la docencia presencial por motivo de la covid-19. En este trabajo analizaremos 4 ítems, 3 de ellos de respuesta abierta y 1 de escala Likert. En los dos primeros se solicitaba a los profesores en formación 5 personajes y 5 acontecimientos relevantes de las transiciones a la democracia en sus respectivos países entre los que debían elegir los 2 que considerasen más importantes. El tercer ítem solicitaba una narrativa con los argumentos que utilizarían en las aulas de educación secundaria para enseñar las transiciones a la democracia. Finalmente, se pedía que indicasen a través de una escala Likert el grado de acuerdo con cuatro perspectivas adaptadas de Kelly (1986) sobre la enseñanza de las transiciones a la democracia caracterizadas como "pasados difíciles" o controvertidos.

Para analizar la estructura de las representaciones de los profesores en 
formación sobre las transiciones a la democracia, se siguieron los criterios de la "teoría del núcleo central" (Abric, 2001, Moliner y Abric, 2015) según la cual las representaciones sociales constan de un núcleo central y una periferia. El núcleo central está formado por elementos que otorgan sentido al conjunto de la representación y son muy estables e independientes de los contextos de los individuos. La periferia está formada por los elementos más variables que se adaptan a diferentes contextos y actúan como protectores del núcleo central. De este modo, analizando los personajes y los acontecimientos citados por los profesores en formación, hemos reconstruido la estructura de sus representaciones sobre la transición dictadura-democracia que influyen en las decisiones sobre los contenidos y las prácticas de enseñanza.

Para el análisis de las narrativas de los profesores en formación sobre las transiciones a la democracia, se han utilizado las categorías de Seixas (2000) que identifica tres tipos de narrativas históricas con funciones epistemológicas y educativas diferentes: memoria colectiva, disciplinar y posmoderna.

Para el análisis de las actitudes de los docentes en formación hacia la enseñanza de "pasados difíciles" o conflictivos, como las transiciones de dictaduras a sistemas democráticos, se adaptaron las categorías de Kelly (1986) que identifica cuatro perspectivas. La denominada neutralidad excluyente propone evitar los temas controvertidos por la imposibilidad de neutralidad del profesor; la parcialidad excluyente propone enseñar una determinada versión excluyendo las demás; la imparcialidad neutral propone enseñar las diferentes versiones, pero sin que el profesor exprese su punto de vista; finalmente, la imparcialidad comprometida propone enseñar las diferentes versiones incorporando las perspectivas y emociones de los estudiantes y del profesor. También resultaron útiles las aportaciones de Zembylas (2018) sobre la resistencia de los profesores a abordar temas conflictivos y la influencia de las emociones en su enseñanza.

Para el tratamiento de los datos se ha utilizado la herramienta Atlas.ti, un software inspirado en la Grounded Theory que proporciona rigor y coherencia al análisis de datos cualitativos, además de facilitar la recuperación y codificación de la información (San Martín, 2014). Se realizaron los tres tipos de codificación de los datos (directa, axial y selectiva), que proponen Strauss y Corbin (2002) para el desarrollo de la Grounded Theory. Para estas tareas, se utilizó la herramienta de Atlas.ti denominada "contador de palabras" y se exportaron los resultados en CSV (Comma Separated Values) para facilitar el proceso de construcción de los gráficos.

\section{Resultados}

A continuación, se presentan los resultados que proporcionan los profesores en formación de España y Portugal referidos a tres aspectos clave: personajes y acontecimientos de las transiciones española y portuguesa, narrativas sobre las 
transiciones que se pretende llevar a las aulas de educación secundaria, y actitudes del profesorado ante la enseñanza de temas históricos conflictivos como las transiciones. Los datos se analizarán haciendo comparaciones entre los resultados de ambos países para identificar semejanzas y diferencias. Para facilitar la lectura de las tablas no se refleja la totalidad de las respuestas, solo las más frecuentes.

\section{Personajes de la transición política en España y Portugal}

Los participantes respondieron de forma adecuada al ítem que solicitaba cinco personajes de las transiciones española y portuguesa, excepto dos estudiantes españoles y tres portugueses que solo proporcionaron tres o cuatro respuestas. Entre los profesores españoles en formación se produjeron 33 variedades de respuesta, mientras los portugueses ofrecieron 16 variedades. En las tablas 1 y 2 se ofrece la nómina de personajes de la transición española y portuguesa. Al final de las tablas también se ofrece la selección de los personajes a los que los participantes otorgan más relevancia (criterio de relevancia en 2 niveles). La frecuencia y la relevancia asignada serán los criterios que delimiten el núcleo central y la periferia de las representaciones.

\section{Tabla 1}

Personajes de la transición política española ( $n=23)$

\begin{tabular}{|c|c|c|}
\hline Personajes & Frecuencia & Porcentaje \\
\hline Adolfo Suárez & 19 & $16,67 \%$ \\
\hline Juan Carlos I & 16 & $14,04 \%$ \\
\hline Felipe González & 9 & $7,89 \%$ \\
\hline Grupos violentos (ETA, GRAPO, GAL) & 8 & $7.05 \%$ \\
\hline Santiago Carrillo & 7 & $6,14 \%$ \\
\hline Sociedad civil & 7 & $6,14 \%$ \\
\hline Manuel Fraga & 5 & $4,39 \%$ \\
\hline Franco & 4 & $3,51 \%$ \\
\hline Arias Navarro & 3 & $2,63 \%$ \\
\hline Oposición al franquismo (externa, interna) & 3 & $2,63 \%$ \\
\hline Calvo Sotelo & 2 & $1.75 \%$ \\
\hline PCE & 2 & $1.75 \%$ \\
\hline Partidos políticos & 2 & $1.75 \%$ \\
\hline Reformistas dentro del franquismo & 2 & $1.75 \%$ \\
\hline Movimientos sociales (obrero, estudiantil, vecinal) & 2 & $1.75 \%$ \\
\hline \multicolumn{3}{|c|}{ Personajes de la transición española. 1ำ nivel de relevancia adjudicada } \\
\hline Adolfo Suárez & 9 & $39,13 \%$ \\
\hline Franco & 2 & $8,70 \%$ \\
\hline \multicolumn{3}{|c|}{ Personajes de la transición española. $2^{\circ}$ nivel de relevancia adjudicada } \\
\hline Juan Carlos I & 5 & $20,00 \%$ \\
\hline Adolfo Suárez & 3 & $12,00 \%$ \\
\hline Felipe González & 2 & $8,00 \%$ \\
\hline
\end{tabular}


Los datos indican que la transición española es vista por los docentes en formación como un proceso protagonizado por dos figuras políticas sobresalientes: el presidente Adolfo Suárez (19 menciones) y el rey Juan Carlos I (16) que aparecen ampliamente destacados de los demás. En un segundo nivel de frecuencia aparecen líderes políticos de diferentes formaciones y con responsabilidades de gestión durante la transición, como Felipe González, Santiago Carrillo, Manuel Fraga, Arias Navarro, Calvo Sotelo y, por supuesto, Franco. Con un nivel de frecuencia más bajo aparecen personajes colectivos, como los grupos violentos, la sociedad civil o los partidos políticos.

Los resultados de los dos personajes que consideran más relevantes dentro de la transición vienen a confirmar lo que ya indican las frecuencias: el núcleo central de las representaciones sobre la transición está compuesto por Adolfo Suárez y Juan Carlos I. Esta representación de la transición española dominada por las élites políticas participa de algunos rasgos de la historiografía tradicional sobre la Transición, y también es fruto de las políticas de memoria desarrolladas durante las últimas décadas a través de los medios de comunicación y las series televisivas que elevaron a ciertos personajes a la categoría de héroes o mitos de la transición. No obstante, la presencia en un segundo lugar de personajes colectivos como los grupos violentos, la sociedad civil o los partidos políticos, ponen de manifiesto que el profesorado en formación está familiarizado con los enfoques historiográficos más recientes que cuestionan los mitos de la transición.

\section{Tabla 2}

Personajes de la transición política portuguesa (n=13)

\begin{tabular}{|c|c|c|}
\hline Personajes & Frecuencia & Porcentaje \\
\hline Mário Soares & 9 & $14,52 \%$ \\
\hline Salgueiro Maia & 9 & $14,52 \%$ \\
\hline Ramalho Eanes & 8 & $12,90 \%$ \\
\hline Álvaro Cunhal & 7 & $11,29 \%$ \\
\hline Antonio de Spínola & 7 & $8,06 \%$ \\
\hline Otelo Saraiva de Carvalho & 6 & $9,68 \%$ \\
\hline Humberto Delgado & 4 & $6,45 \%$ \\
\hline Sá Carneiro & 3 & $4,84 \%$ \\
\hline Freitas do Amaral & 2 & $3,23 \%$ \\
\hline Capitanes de Abril & 1 & $1,61 \%$ \\
\hline Zeca Afonso & 1 & $1,61 \%$ \\
\hline Vasco Gonzalves & 1 & $1,61 \%$ \\
\hline Quintão Meireles & 1 & $1,61 \%$ \\
\hline \multicolumn{3}{|c|}{ Personajes de la transición portuguesa. 1 nivel de relevancia adjudicada } \\
\hline Salgueiro Maia & 3 & $25,00 \%$ \\
\hline Álvaro Cunhal & 2 & $16,67 \%$ \\
\hline Humberto Delgado & 2 & $16,67 \%$ \\
\hline Mário Soares & 2 & $16,67 \%$ \\
\hline
\end{tabular}

Personajes de la transición portuguesa. $2^{\circ}$ nivel de relevancia adjudicada 
La transición portuguesa según los docentes en formación está asociada a varios personajes individuales procedentes tanto del sector militar como del político, entre los que destacan Mario Soares y Salgueiro Maia (9 menciones), Ramalho Eanes (8), Alvaro Cunhal o Antonio de Spínola (7) y Otelo Saraiva de Carvalho (6). Al lado de estos protagonistas individuales tan solo aparece un personaje colectivo, capitanes de abril, el grupo de militares del Movimiento de las Fuerzas Armadas que protagonizó la revolución. Con frecuencias menores aparecen otros personajes entre los que señalamos a Zeca Afonso, el autor de la canción "Grándola" que señaló el inicio de la revolución.

Los docentes portugueses parecen repartir el protagonismo de la transición de su país entre un número mayor de personajes que en el caso español. Teniendo en cuenta los niveles de relevancia de los personajes al final de la tabla 2, se confirma que Mario Soares y Salgueiro Maia son las figuras que gozan de mayor valoración; son, por lo tanto, las que ocupan el lugar central dentro de las representaciones del profesorado portugués en formación, al tiempo que representan de forma paradigmática a los sectores político y militar de la transición. Como en el caso español, esta representación se elabora a partir de las aportaciones de la historiografía, pero también de las políticas de memoria que los medios y el cine han contribuido a difundir.

\section{Acontecimientos de la transición política en España y Portugal.}

En respuesta al ítem que solicitaba acontecimientos de las transiciones, los participantes españoles proporcionaron 34 acontecimientos diferentes y los portugueses 20. En las tablas 3 y 4 se ofrecen las respuestas con el número de menciones (criterio de frecuencia), así como los acontecimientos a los que conceden mayor relevancia (criterio de relevancia).

\section{Tabla 3}

Acontecimientos de la transición política española $(n=23)$

\begin{tabular}{lc}
\hline Acontecimientos & Frecuencia \\
\hline Constitución de1978 & 17 \\
\hline Golpe del 23F & 13 \\
\hline Muerte de Franco & 11 \\
\hline Primeras elecciones democráticas & 11 \\
\hline Estatutos de Autonomía & 5 \\
\hline Ley de amnistía & 5 \\
\hline Legalización del PCE & 5 \\
\hline Atentados de Atocha & 4 \\
\hline Pactos de la Moncloa & 4 \\
\hline
\end{tabular}


Las transiciones a la democracia en España y Portugal como pasados difíciles. Representaciones sociales del profesorado de Educación Secundaria en formación

\begin{tabular}{lc} 
Atentado de Carrero Blanco & 3 \\
\hline Restablecimiento de la monarquía & 3 \\
\hline Crisis del Sahara & 2 \\
\hline Ley de partidos & 2 \\
\hline Movimientos sociales (obrero, estudiantil, vecinal) & 2 \\
\hline Victoria de Adolfo Suárez & 2 \\
\hline \multicolumn{1}{c}{ Acontecimientos de la transición española. 1o nivel de relevancia adjudicada } \\
\hline Constitución_1978 & 6 \\
\hline Muerte de Franco & 5 \\
\hline
\end{tabular}

Los profesores españoles en formación seleccionan cuatro acontecimientos ampliamente destacados del resto: la constitución de 1978, el fallido golpe de estado de 1981, la muerte de Franco y las primeras elecciones democráticas; unos acontecimientos que hacen referencia, por una parte, al final de la dictadura y las resistencias a su desaparición y, por otra, a las manifestaciones más emblemáticas del sistema democrático. Si estos acontecimientos hacen referencia a aspectos políticos con un elevado valor simbólico que vuelven a traer al primer plano una visión complaciente de la transición, otros con un menor número de menciones revelan visiones más críticas y más acordes con las corrientes historiográficas recientes, como la ley de amnistía, los pactos de la Moncloa, la forma monárquica de gobierno, la violencia persistente de diferente signo (atentados de Carrero Blanco y de Atocha) o el papel de los movimientos sociales.

El criterio de relevancia otorgada por los participantes a los acontecimientos confirma nuevamente lo que indican las frecuencias y sitúa la constitución de 1978 y la muerte del dictador en el núcleo central de las representaciones.

\section{Tabla 4}

Acontecimientos de la transición política portuguesa $(n=11)$

\begin{tabular}{lc}
\hline \multicolumn{1}{c}{ Acontecimientos } & Frecuencia \\
\hline Revolución del 25 de abril & 9 \\
\hline Constitución y elecciones de 1976 & 5 \\
\hline Guerra colonial y descolonización & 5 \\
\hline Golpe de 25 de noviembre de 1975 & 4 \\
\hline Adhesión a la CEE & 2 \\
\hline Candidatura/acciones de Humberto Delgado & 2 \\
\hline Creación del MFA & 2 \\
\hline Elecciones Presidenciales de 1958 & 2 \\
\hline Fuerte presencia del partido comunista & 1 \\
\hline Gobierno de Marcelo Caetano & 1 \\
\hline Muerte de Salazar & 1 \\
\hline Exilio de personalidades destacadas de la dictadura & 1 \\
\hline Golpe del 11 de marzo de 1975 & 1 \\
\hline
\end{tabular}

Acontecimientos de la transición portuguesa. 1 nivel de relevancia adjudicada Revolución del 25 de abril de 1974

Guerra colonial 
Los profesores portugueses en formación citan cuatro acontecimientos con un mayor número de menciones: la revolución del 25 de abril, destacado a gran distancia del resto, la constitución de 1976, la guerra colonial y el golpe del 25 de noviembre de 1975. Estos acontecimientos ponen de manifiesto la particularidad revolucionaria de la transición portuguesa y la influencia que la guerra colonial tuvo como desencadenante del Movimiento de la Fuerzas Armadas y de la revolución; por lo demás, las semejanzas con los participantes españoles se refieren al valor simbólico de la constitución, al camino incierto de la transición a la democracia y los intentos golpistas de contenerla o hacerla fracasar. La muerte del dictador, que en el caso español aparece en un lugar muy destacado, ocupa aquí un espacio irrelevante, poniendo de manifiesto las diferencias entre la vía revolucionaria portuguesa y la reformista española.

Si reparamos en la relevancia adjudicada a los acontecimientos, la revolución del 25 de abril y la guerra colonial ocupan el núcleo central de las representaciones, si bien la primera ocupa la mayor parte de ese espacio. De hecho, estos dos eventos han dejado una huella duradera en la sociedad portuguesa de hoy.

\section{Narrativas de los docentes españoles y portugueses en formación sobre la enseñanza de las transiciones.}

Las respuestas de los participantes al ítem en que se solicitaba la selección de las ideas clave para articular la narrativa de las transiciones, permiten identificar el tipo de narrativa histórica que los profesores en formación pretenden llevar a las aulas y la función educativa que puede cumplir (Seixas, 2000). En las figuras 1 y 2 se ofrecen las ideas presentes en las narrativas, ordenadas por la frecuencia de aparición. 


\section{Figura 1}

Ideas clave de los docentes en formación sobre la transición española para trabajar en las aulas de educación secundaria. Elaboración propia

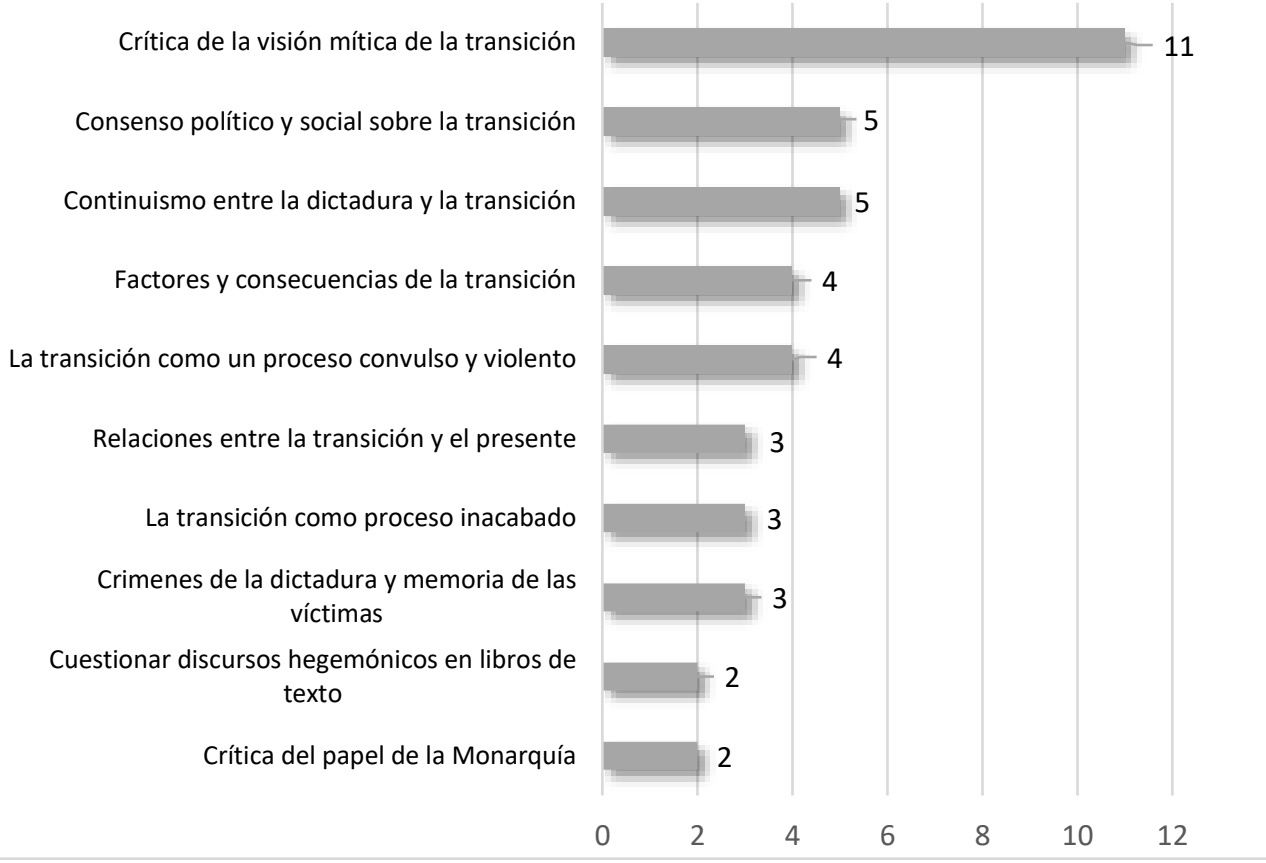

La enseñanza de la transición según los profesores españoles en formación debe cumplir una función fundamental: contribuir a depurar la visión complaciente y mítica que la historiografía tradicional, las políticas de memoria y los medios de comunicación han transmitido. En este mismo sentido de apostar por utilizar los conceptos y métodos de la disciplina (perspectiva disciplinar) para cuestionar las tradiciones y producir conocimiento riguroso apuntan ideas como el continuismo de personas e instituciones de la dictadura, el carácter violento y convulso de la transición frente a la visión idealizada y pacífica, o los crímenes de la dictadura ocultados por una cierta política de blanqueado del franquismo. También apuntan en esta dirección las propuestas de incidir sobre los factores y las consecuencias de la transición o la crítica del conocimiento estereotipado que se transmite con frecuencia a través de los libros de texto.

Algunas otras ideas parecen corresponder con las que Seixas (2000) denomina narrativas posmodernas, que tratan de desvelar los usos que diferentes grupos sociales hacen del pasado en las sociedades actuales. En esta dirección apuntan aspectos como las relaciones entre la transición y el presente o la transición como un proceso inacabado que todavía plantea retos a la sociedad actual. 
Estas tipologías narrativas no son compartimentos estancos ni excluyentes, de forma que los profesores frecuentemente participan de varios de estos enfoques. En esta dirección parece apuntar uno de los aspectos señalados por los participantes cuando hacen referencia al consenso político y social sobre la transición. Esta idea podría asociarse con la perspectiva memoria colectiva y que trata de transmitir un discurso consensual sobre el pasado (en este caso, la transición). Esta idea entraría en contradicción con los diferentes proyectos políticos presentes en la transición o con el carácter violento y convulso de la misma.

\section{Figura 2}

Ideas clave de los docentes en formación sobre la transición portuguesa para trabajar en las aulas de educación secundaria. Elaboración propia

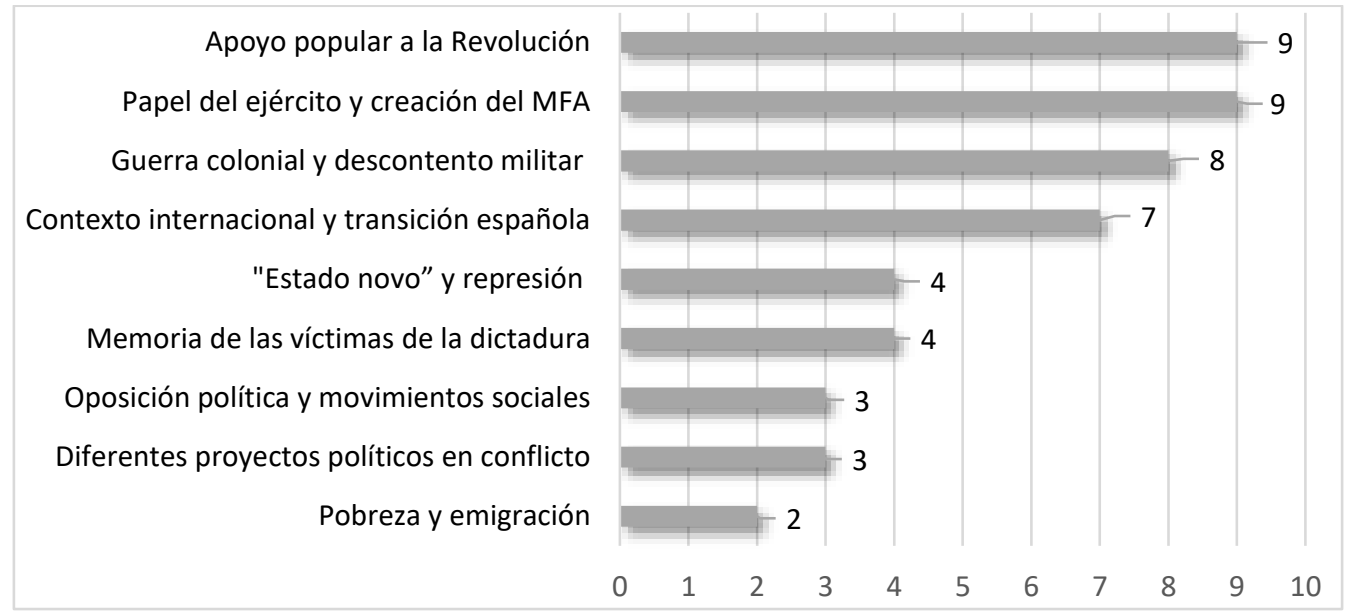

La narrativa de los profesores portugueses en formación se articula desde una perspectiva disciplinar en torno a un proceso revolucionario protagonizado por el Movimiento de las Fuerzas Armadas, con un fuerte apoyo popular y en el que la guerra colonial y el descontento militar actuaron como desencadenantes; todo ello en un contexto internacional en el que la transición española también acabó influyendo. Otros aspectos como la relevancia de la represión del Estado Novo, la memoria de las víctimas o la diversidad de proyectos políticos en conflicto parecen apuntar en la dirección del enfoque posmoderno al tratar de poner de manifiesto diferentes narrativas sobre la transición con diferentes efectos sobre el presente. Tampoco está ausente el enfoque de memoria colectiva en un cierto carácter consensual sobre el papel del ejército o el apoyo popular a la revolución, ocultando los enfrentamientos entre las diferentes facciones del ejército que terminaron con la disolución del Movimiento de las Fuerzas Armadas, o los sucesivos golpes para conducir la revolución hacia la consolidación del régimen de la transición. 


\section{Actitudes de los profesores en formación ante la enseñanza de las transiciones en España y Portugal.}

En el último ítem que analizaremos se solicitaba a los participantes que expresasen a través de una escala Likert su grado de acuerdo con cuatro actitudes de los profesores a la hora de trabajar en las aulas con pasados difíciles o controvertidos, como las transiciones española y portuguesa. Las cuatro actitudes se han adaptado a partir de Kelly (1986). En las tablas 5 y 6 aparecen los grados de acuerdo de los participantes españoles y portugueses con estas cuatro perspectivas.

\section{Tabla 5}

Actitudes de los profesores españoles en formación sobre la enseñanza de las transiciones $(n=24)$

\begin{tabular}{lccccc}
\hline Escala & $\begin{array}{c}\text { totalmente } \\
\text { en } \\
\text { Actitudes }\end{array}$ & $\begin{array}{c}\text { muy en } \\
\text { desacuerdo }\end{array}$ & $\begin{array}{c}\text { algo de } \\
\text { acuerdo }\end{array}$ & $\begin{array}{c}\text { muy de } \\
\text { acuerdo }\end{array}$ & $\begin{array}{c}\text { totalmente de } \\
\text { acuerdo }\end{array}$ \\
\hline $\begin{array}{l}\text { Neutralidad } \\
\text { excluyente }\end{array}$ & 24 & 0 & 0 & 0 & 0 \\
\hline $\begin{array}{l}\text { Parcialidad } \\
\text { excluyente }\end{array}$ & 17 & 6 & 0 & 1 & 0 \\
\hline $\begin{array}{l}\text { Imparcialidad } \\
\text { neutral }\end{array}$ & 2 & 8 & 7 & 4 & 3 \\
\hline $\begin{array}{l}\text { Imparcialidad } \\
\text { comprometida }\end{array}$ & 0 & 3 & 4 & 10 & 7
\end{tabular}

Los profesores españoles en formación creen que la actitud denominada imparcialidad comprometida es la más deseable ya que 17 de los 24 se muestran totalmente de acuerdo o muy de acuerdo con esta perspectiva y ninguno se manifiesta en total desacuerdo. Menos adhesión consigue la perspectiva denominada imparcialidad neutral ya que son 7 participantes los que se muestran totalmente de acuerdo o muy de acuerdo, pero otros 10 se muestran muy en desacuerdo o totalmente en desacuerdo. En el extremo contrario, la totalidad de los participantes rechazan la perspectiva de la neutralidad excluyente; a su vez, 23 e los 24 participantes se muestran en total desacuerdo o muy en desacuerdo con la perspectiva parcialidad excluyente. Es posible que esta opción tan mayoritaria a favor de la imparcialidad neutral y comprometida venga influida por el hecho de que estas perspectivas fueron conocidas por los estudiantes en el programa formativo. 


\section{Tabla 6}

Actitudes de los profesores portugueses en formación sobre la enseñanza de las transiciones $(n=15)$

\begin{tabular}{lccccc}
\hline \multicolumn{1}{c}{ Escala } & $\begin{array}{c}\text { totalmente } \\
\text { en } \\
\text { desacuerdo }\end{array}$ & $\begin{array}{c}\text { muy en } \\
\text { desacuerdo }\end{array}$ & $\begin{array}{c}\text { algo de } \\
\text { acuerdo }\end{array}$ & $\begin{array}{c}\text { muy de } \\
\text { acuerdo }\end{array}$ & $\begin{array}{c}\text { totalmente } \\
\text { de acuerdo }\end{array}$ \\
\hline $\begin{array}{l}\text { Neutralidad } \\
\text { excluyente }\end{array}$ & 13 & 2 & 0 & 0 & 0 \\
\hline $\begin{array}{l}\text { Parcialidad } \\
\text { excluyente }\end{array}$ & 11 & 4 & 0 & 0 & 8 \\
\hline $\begin{array}{l}\text { Imparcialidad } \\
\text { neutral }\end{array}$ & 0 & 2 & 1 & 4 & 3 \\
\hline $\begin{array}{l}\text { Imparcialidad } \\
\text { comprometida }\end{array}$ & 2 & 1 & 5 & 4 &
\end{tabular}

Los docentes portugueses en formación muestran un rechazo semejante a los españoles hacia las actitudes neutralidad y parcialidad excluyentes ya que ninguno se sitúa en los valores positivos de estas opciones. La perspectiva que cuenta con mayor aceptación es la de la imparcialidad neutral ya que 12 de los 15 participantes se muestran totalmente de acuerdo o muy de acuerdo; por su parte, respecto a la imparcialidad comprometida solo se sitúan en los valores más elevados 7 de los 15 participantes, unos resultados inferiores a los de los participantes españoles que podrían estar en relación con el hecho ya señalado de que estas actitudes hacia la enseñanza de los temas controvertidos resultasen más familiares para los participantes españoles que para los portugueses en sus procesos de formación.

\section{Conclusiones}

El estudio ha permitido aproximarse a las representaciones de una muestra de estudiantes de Máster en Profesorado de Educación Secundaria de España y Portugal sobre las transiciones en sus respectivos países y los problemas de su enseñanza como pasados difíciles o controvertidos.

El análisis de la estructura de las representaciones sociales del profesorado en formación siguiendo la teoría del núcleo central (Abric, 2001, Moliner y Abric, 2015) pone de manifiesto que los estudiantes españoles perciben el proceso de transición protagonizado por sectores de las élites políticas (Adolfo Suárez y Juan Carlos I ocupan el núcleo central de las representaciones), una visión construida a partir de aportaciones de la historiografía tradicional, los medios de comunicación y las series de televisión. En el terreno de los acontecimientos, esta visión más tradicional y complaciente estaría representada por acontecimientos exitosos como las primeras elecciones democráticas y la constitución de 1978. Pero, al lado de esta visión más tradicional, aparecen personajes colectivos, como los grupos violentos, 
la sociedad civil o los partidos políticos, y acontecimientos como el golpe del 23F, los atentados de Atocha o la ley de amnistía que hacen referencia al carácter convulso y violento de la transición (frente a la visión oficial de modelo de transición pacífica), y a aspectos como la amnistía a los represores de la dictadura o la institución monárquica que son objeto de amplios debates en la sociedad actual y por las que se interesa la nueva historiografía de la transición (Baby, 2015; Sánchez y Aguilar, 2009; Ysás, 2010).

El profesorado portugués en formación concibe la revolución y la transición a la democracia como la acción de una serie de líderes políticos y militares, entre los que Mario Soares y Salgueiro Maia ocupan el núcleo central de las representaciones, acompañados por un personaje colectivo, los "capitanes de abril", que representan la iniciativa del Movimiento de las Fuerzas Armadas en la revolución de los claveles. Entre los acontecimientos más destacados, la revolución del 25 de abril y la guerra colonial ocupan el núcleo central de las representaciones, poniendo de manifiesto la particularidad de la vía revolucionaria de transición portuguesa a la democracia.

El análisis de las narrativas que el profesorado en formación elabora para trabajar las transiciones en las aulas de secundaria pone de manifiesto que el tipo de narrativa que Seixas (2000) denomina disciplinar es la que obtiene más acogida tanto entre los participantes españoles como portugueses. Una narrativa que apuesta por utilizar las herramientas conceptuales y metodológicas de la historia para poner en cuestión las visiones complacientes o románticas de las transiciones difundidas a través de la historiografía tradicional y los medios de comunicación. Estas narrativas disciplinares ponen de manifiesto la diversidad de proyectos sociales presentes en el momento de la crisis de las dictaduras, los crímenes de las dictaduras que ciertas políticas de blanqueado han tratado de minimizar, o el carácter convulso e incierto de las transiciones. Algunas otras ideas parecen corresponderse con la tipología narrativa posmoderna, que pretende desvelar los usos del pasado por los diferentes grupos sociales en las sociedades actuales. Esta tipología narrativa se manifiesta, por ejemplo, en la atención que los participantes prestan a la memoria de las víctimas de las dictaduras o a la diversidad de proyectos sociales presentes en los dos procesos de transición. Pero, dado que estas narrativas non son excluyentes, algunas ideas como el consenso social sobre la transición pacífica española o el apoyo popular a la revolución portuguesa parecen corresponderse con la tipología narrativa de memoria colectiva, que trata de crear consenso y cohesión social entorno a un determinado resultado de los procesos de transición.

Por último, el análisis de las actitudes del profesorado en formación respecto a la enseñanza de las transiciones como pasados difíciles o controvertidos revela que, tanto los participantes españoles como portugueses, rechazan las perspectivas definidas por Kelly (1986) como neutralidad excluyente y parcialidad excluyente, que evitan o proporcionan una perspectiva parcial de la transición en las aulas. El profesorado en formación español se inclina mayoritariamente por la perspectiva imparcialidad comprometida que propone hacer presentes en las aulas las 
diferentes versiones de los acontecimientos incorporando los puntos de vista y las emociones de alumnado y profesorado. Por su parte, el profesorado portugués en formación parece inclinarse más hacia la perspectiva denominada imparcialidad neutral, que propone ofrecer las diferentes versiones de los acontecimientos conflictivos evitando el punto de vista del profesor para no influir en el alumnado.

El trabajo se realiza con una muestra reducida de profesorado en formación que deberá ampliarse en estudios futuros, pero los datos proporcionan interpretaciones coherentes con trabajos anteriores sobre las perspectivas del profesorado en relación a las transiciones (Martínez, 2013; Martínez, Muñoz y Sánchez, 2019; Santiago, 2014). También pone de manifiesto semejanzas y diferencias entre los participantes españoles y portugueses consistentes con diferentes procesos formativos.

Abordar la enseñanza de temas históricos conflictivos, como las transiciones de la dictadura a la democracia, en los programas de formación del profesorado de historia y ciencias sociales debe contribuir, por una parte, a desvelar la influencia de los medios de comunicación y las políticas de memoria en la pervivencia de ciertas perspectivas tradicionales y mitificadas de las transiciones e identificar las perspectivas historiográficas más recientes sobre los procesos de transición; por otra parte, debe poner de manifiesto las diversas memorias y los debates existentes en la sociedad actual sobre el significado de las transiciones a la democracia, así como las emociones que acompañan a estos debates; por último, debe crear oportunidades para que el profesorado en formación analice y valore propuestas de enseñanza al mismo tiempo que revisa las propias emociones, resistencias y actitudes hacia la enseñanza de estas temáticas conflictivas de la historia reciente.

\section{Referencias}

Abric, J.C. (Dir.) (2001). Prácticas sociales y representaciones. Ediciones de Coyoacán.

Aceituno, D. (2011). Transición y democracia: algunos datos sobre manuales escolares en 4ํ de la ESO. En, R. López, L. Velasco, V. Santidrián y X. Armas (Eds.). Pensar históricamente en tiempos de globalización (pp. 15-28). Universidad de Santiago de Compostela

Alves, L. A., Ribeiro, C., Oliveira, R., y Moreira, L. (2012). Ideias de alunos sobre o "seu" passado doloroso a Guerra Colonial Portuguesa. Em Tempo de Histórias, 21, 7-31.

Armas, J., Moreira, A.I., Maia, C. y Conde, J. (2019). La historia de Portugal en las aulas de Educación Básica ¿Formar patriotas o educar ciudadanos? Revista Electrónica Interuniversitaria de Formación del Profesorado, 22(2), 67-80.

Baby, S. (2015). Volver sobre la Inmaculada Transición. El mito de una transición pacífica en España. En M. C. Chaput y J. Pérez Serrano (Eds.). La 
transición española. Nuevos enfoques para un viejo debate (pp. 75-92). Biblioteca Nueva.

Baby, S., Compagnon, O., y González, E. (Dirs.) (2009). Violencia y transiciones políticas a finales del siglo XX. Europa del Sur y América Latina. Casa de Velázquez.

Barca, I., y Schmidt, M. A. (2013). La consciencia histórica de los jóvenes brasileños y portugueses y su relación con la creación de identidades nacionales. Educatio Siglo XXI, 31(1), 25-46.

Barton, K. C. (2019). Teaching Difficult Histories. The Need for a Dynamic Research Tradition. En M. H. Gross and L. Terra (Eds.) (2019). Teaching and Learning the Difficult Pasts. Comparative Perspectives (pp. 11-25). Routledge

Castorina, J. A., y Barreiro, A. (2012). Los usos de las representaciones sociales en la investigación educativa. Educación, Lenguaje y Sociedad, IX(9), 1540 .

Chaput, M. C., y Pérez Serrano, J. (Eds.) (2015). La transición española. Nuevos enfoques para un viejo debate. Madrid, Biblioteca Nueva.

Denzin, N. K., y Lincoln, Y. S. (Eds.) (2018). The SAGE Handbook of Qualitative Research. Sage.

Epstein, T., y Peck, C. L. (Eds.) (2018). Teaching and Learning Difficult Histories in International Contexts. A Critical Sociocultural Approach. Routledge.

Feria, P. (2018). Del Mediterráneo al Cono Sur: las transiciones a la democracia de Chile, Portugal y España en perspectiva comparada. Revista de Historia Social y de las Mentalidades, 22(2), 103-135.

Férias, A., Armas, X. y Maia, C. (2019). Histórias difíceis e sensíveis no 1. Ciclo do Ensino Básico em Portugal. Currículo, salas de aula e espaços públicos. Comunicação Oral apresentada no Conferência Internacional de Educação Histórica e Patrimonial do Politécnico do Porto. Porto (na imprensa).

Flick, U. (2015). El diseño de la investigación cualitativa. Morata.

Goldberg, T., and Savenije, G. M. (2018). Teaching Controversial Historical Issues. En S. A. Metzger and L. M. Harris (eds.) The Wiley International Handbook of History Teaching and Learning (pp. 503-526). John Wiley \& Sons.

Gómez, A. (2011). La llegada de la democracia al Mediterráneo: las transiciones de Portugal, Grecia y España. Historia Actual Online, 25, 7-18.

Gross, M, y Terra, L. (2018). What Makes Difficult History Difficult? Phi Delta Kappan, 99(8), 51-56.

Gross, M., y Terra, L. (Eds.) (2019). Teaching and Learning Difficult Pasts: Comparative Perspectives. Routledge. 
Izquierdo, J. (2018). Recuerdo sobre fondo gris. Democracia y memoria herida en España. CONFLUENZE. Revista di Studi Iberoamericani, X (2), 105-126. DOI: $10.6092 /$ issn.2036-0967/8884.

Jodelet, D. (2011). Aportes del enfoque de las representaciones sociales al campo de la educación. Espacios en blanco. Serie indagaciones, 21(1), 133154.

Kelly, T. E. (1986). Discussing Controversial Issues: Four Perspectives on the Teacher's Role. Theory and Research in Social Education, XIV (2), 113-138.

Lemus, E. (2012). La Transición Ibérica (Presentación). HISPANIA. Revista Española de Historia, LXXII (242), 635-638.

Loff, M. (2004). ¿Revolución versus transición? Visiones de España desde el Portugal revolucionario y postrevolucionario. Gerónimo de Uztariz, 20, 17 44.

Loff, M., Soutelo, L., y Piedade, F. (Coords.) (2014). Ditaduras e Revolução. Democracia e políticas de memória. Ediciones Almeida

Martínez, R. (2011) Historia y formación ciudadana. Un análisis a través de la transición a la democracia en los manuales de Educación para la Ciudadanía. En R. López, L. Velasco, V. Santidrián y X. Armas (Eds.). Pensar históricamente en tiempos de globalización (pp. 125-138). Universidad de Santiago de Compostela.

Martínez, R. (2013). Profesores entre la historia y la memoria. Un estudio sobre la enseñanza de la transición dictadura-democracia en España (Tesis doctoral). Universidad de Valladolid. Disponible en https://uvadoc.uva.es/bitstream/handle/10324/4100/TESIS410131213.pdf

Martínez, R., Muñoz, C., Sánchez, M. (2019). Conocimientos y creencias del profesorado y su vínculo con las finalidades de la enseñanza de la historia reciente. La transición a la democracia en España como caso controvertido. Revista de Educación, 383, 11-35. doi: 10.4438/1988-592XRE-2019-383-399.

Moliner, P., y Abric, J. C. (2015). Central core theory. En G. Sammut, E. Andreouli, G. Gaskell, y J. Valsiner (Eds.) The Cambridge Handbook of Social Representations (pp. 83-95). Cambridge University Press.

Molpeceres, A. (2011). Conflictos: la memoria de los alumnos. De la Guerra Civil a la transición. Iber: Didáctica de las ciencias sociales, geografía e historia, 69, 21-28.

Moscovici, S. (2000). Social Representations. Explorations in Social Psychology. Polity Press.

Sammut, G., Andreouli, E., Gaskell, G., y Valsiner, J. (Eds.) (2015). The Cambridge Handbook of Social Representations. Cambridge University Press. 
San Martín, D. (2014). Teoría fundamentada y Atlas. ti: recursos metodológicos para la investigación educativa. Revista electrónica de investigación educativa, 16(1), 104-122. Recuperado de http://redie.uabc.mx/vol16no1/contenido-sanmartin.html.

Sánchez, I., y Aguilar, P (2009). Violencia política y movilización social en la transición española. En S. Baby, O. Compagnon, y E. González (Dirs.). Violencia y transiciones políticas a finales del siglo XX. Europa del Sur y América Latina (pp. 95-111). Casa de Velázquez.

Sánchez, M., Martínez, R., Miguel D., y López, E. (2019). Ideas de los jóvenes españoles acerca del pasado reciente: el caso de la transición a la democracia. El Futuro del Pasado, 10, 215-255. doi: 10.14516/fdp.2019.010.001.008.

Santiago, B. (2014). La Transición española en 4. ESO. Un estudio de caso de los significados. Panta Rei. Revista Digital de Ciencia y Didáctica de la Historia, 121-132.

Schmitter, Ph., y Lynn, K. (1994). La transitología ¿ciencia o arte de la democratización? En Ph. Schmitter, La consolidación democrática en América Latina. Hacer Editorial.

Seixas, P. (2000). Schweigen! die Kinder! or, Does Postmodern History Have a Place in the Schools? En P. N. Stearns, P. Seixas \& S. Wineburg (Eds.). Knowing, Teaching and Learning History. National and International Perspectives (pp. 19-37). New York University Press.

Soto, A. (2009). La transición a la democracia en el sur de Europa. La historia como instrumento para su comparación. Estudios Internacionales, 162, 7 30 .

Strauss, A., y Corbin, J. (2002) Bases de la investigación cualitativa. Técnicas y procedimientos para desarrollar la Teoría Fundamentada. Universidad de Antioquia.

Varela, R. (Coord.) (2012), Revolução ou Transição. História e Memória da Revolução dos Cravos. Lisboa: Bertrand.

Vásquez, G., Sánchez, M., y Vásquez, N. (2018). Los Hechos Históricos de la Transición Democrática Chilena en las Aulas de Educación Secundaria. Revista História Hoje, 7(13), 106-127.

Ysàs, P. (2010). La Transición española. Luces y sombras. Ayer, 79(3), 31-57.

Zembylas. M. (2018). Teacher Resistance towards Difficult Histories. The Centrality of Affect in Disrupting Teacher Learning. En T. Epstein \& C. L. Peck (Eds.). Teaching and Learning Difficult Histories in International Contexts (pp. 189-200). Routledge 
